\title{
Correlations between magnetic properties and bond formation in $\mathrm{Rh}-\mathrm{MgO}(001)$
}

\author{
Christopher WM Castleton ${ }^{1,2}$, Somkiat Nokbin ${ }^{1,3}$ and Kersti Hermansson ${ }^{1}$ \\ ${ }^{1}$ Materials Chemistry, The Angström Laboratory, Box 538, \\ SE-75121 Uppsala, Sweden \\ ${ }^{2}$ Department of Physics and Mea surement Technology, Linköping University, \\ SE-58183 Linköping, Sweden \\ ${ }^{3}$ Laboratory for Computational and Applied Chemistry, Department of Chemistry, Kasetsart \\ University, Bangkok 10900, Thailand
}

\begin{abstract}
We present the results of first principles calculations for the magnetism of $\mathrm{Rh}$ adlayers on $\mathrm{MgO}$ (001), at three different adsorption sites and three different coverages, corresponding to $1,1 / 2$ and 1/8 monolayers. Finite magnetization is found in all cases except that of $1 \mathrm{Rh}$ monolayer above the oxygen site, which is also the most stable. We examine how the magnetization changes as a function of the Rh-surface distance and relate this to changes in the real space charge density and in the density of states (DOS) as the Rh adlayer interacts with the surface. We find that increasing either the $\mathrm{Rh}-\mathrm{Rh}$ interaction strength or the $\mathrm{Rh}$-surface interaction strength leads to reduced magnetization, while increasing the former drives a crossover from localized (atomic) to itinerant magnetism. Neither the magnetic transition itself, nor the localized-to-itinerant magnetism crossover, is found to be directly related to the formation of $\mathrm{Rh}$ surface bonds. From a practical point of view, we predict that magnetism in the Rh-MgO(001) system is most likely to be found experimentally at reduced coverages and at low temperatures.
\end{abstract}




\section{Introduction}

The study of magnetism in transition metals has a very long history. Most of the transition metals have a finite moment for the isolated atom, but magnetism in the three-dimensional (3D) bulk materials is very rare. Indeed, only four of the metals are magnetic and they are all from the first (3d) transition metal elements, with $\mathrm{Fe}$, Co and $\mathrm{Ni}$ being ferromagnets and $\mathrm{Mn}$ an antiferromagnet. All the other $3 \mathrm{~d}$ and all of the $4 \mathrm{~d}$ and $5 \mathrm{~d}$ transition metal atoms loose their magnetism when they bond to gether to form the bulk metals. However, in recent years there has been a great deal of interest in the possibility of forcing some of them to remain magnetic by reducing the dimensionality below that of the bulk metal. The interest is both fundamental and in the context of possible technological applications in spintronics and in magnetic data storage and retrieval. It has largely centred on the search for ferromagnetism in elements from the upper half of the second (4d) and third (5d) transition metal series. In this paper we will focus on one of the more commonly studied examples, $\mathrm{Rh}$.

$\mathrm{Rh}$ has been shown to display a rich variety of magnetic behaviour as a function of dimensionality. Starting from $O D$ systems: atomic $\mathrm{Rh}$ has the electronic configuration $4 \mathrm{~d}^{8} 5 \mathrm{~s}^{1}$, leading to a ground-state magnetic moment of $3 \mu_{B}$ according to Hund's rules. $0 D \mathrm{Rh}_{n}(\mathrm{n}=9 \Rightarrow 34)$ nano-clusters have been studied experimentally by Cox et al. [1,2], who found moments of $0.4 \Rightarrow 0.8 \mu_{\mathrm{B}}$ for clusters with $\mathrm{n} \leq 20$, dropping to $0.0 \Rightarrow 0.2 \mu_{\mathrm{B}}$ above this size. Various theoretical studies [3-10] predict a rich variety of magnetic properties, with non-magnetic, ferromagnetic and antiferromagnetic ground states of $0 \Rightarrow 2 \mu_{\mathrm{B}}$ all appearing for different values of $\mathrm{n}$. In addition to this, $\mathrm{OD}$ islands of $\mathrm{Rh}$ grown on $\mathrm{Ag}$ substrates have also been predicted to be magnetic [11].

Increasing to $1 D$ systems, Rh chains grown along the $<010>$ and $<110>$ directions on the (001) surface of $\mathrm{Ag}$ have been predicted to be magnetic [11,12]. However, experimental growth of such systems has been found to be very hard to control, so they are currently of limited interest techno logically.

More interesting is the possibility of keeping Rh magnetic in 2D systems [13]. The most obvious 2D system is actually the surface of bulk Rh crystals, and spin-dependent photoemission [14] does indeed find a weak magnetization of $0.2 \mu_{\mathrm{B}}$, in agreement with theoretical predictions [15]. More "strictly" 2D systems have also been studied, in the form of $\mathrm{Rh}$ thin films and monolayers (MLs) grown on a variety of substrates. Here, most of the systems examined have been $\mathrm{Rh}$ adlayers on noble metal substrates. Theoretical predictions have suggested that single Rh MLs will be magnetic on $\mathrm{Ag}(111)$ [16,17] and (001) [13,16,18-24], $\mathrm{Au}$ (111) [16] and (001) [13,16,23,25], Ni (111) [26] and $\mathrm{Cu}(001)$ [16,27-29] but non-magnetic on Ag (110) [16], Au (110) [16], $\mathrm{Cu}(110)$ [16] and $\mathrm{Cu}$ (111) [16]. In the magnetic cases, the moments are predicted to 
decrease rapidly with increasing numbers of Rh layers, usually becoming very weak by film thicknesses of just $2 \mathrm{ML}$ [26,30]. Unfortunately, all attempts to experimentally demonstrate the presence of a magnetic moment in such a system have failed [31-33]. The explanation seems to be that layer-by-layer growth is too difficult to obtain on these substrates, due to problems with lattice constant mismatch, interdiffusion and alloying [29,34]. Theoretical work shows that this should indeed destroy the anticipated magnetism [18,24,35].

The obvious alternative is to study MLs grown on non-metallic substrates. So far one such transition metal system has been found to be magnetic: $\mathrm{Ru}$ grown on $\mathrm{C}(0001)[36,37]$. Experimental studies of Rh MLs on non-metallic substrates have (to our knowledge) yet to be reported, but theoretical predictions suggest that $\mathrm{Rh}$ should remain magnetic on $\mathrm{C}(0001)$ [37-39], albeit weakly. One further non-metallic substrate has been considered theoretically: $\mathrm{MgO}$. Experimentally, $\mathrm{MgO}(001)$ is used frequently as a substrate for metallic thin film growth (see references [40-48], for example). As for the $3 \mathrm{~d}$ metals, Li and Freeman [40] have studied magnetism theoretically in the $\mathrm{Fe} / \mathrm{MgO}(001)$ system. They predicted a very large moment of $3.07 \mu_{\mathrm{B}}$ for Fe, almost the same as that they found for the free Fe ML $\left(3.10 \mu_{\mathrm{B}}\right)$. Moreover, $2 \mathrm{ML}$ of Fe on $\mathrm{MgO}(001)$ has also been predicted to be magnetic, with magnetic moments of 2.85 and $2.96 \mu_{\mathrm{B}}$ for the first and second ML, respectively [40]. Theoretical calculations for various $4 \mathrm{~d}$ transition metals on $\mathrm{MgO}(001)$ have also been reported. Using full-potential linearized augmented-plane-wave (FLAPW) periodic calculations (see Ref. [49] for details) Wu and Freeman [42] found that $1 \mathrm{ML} \mathrm{Ru}$ and $\mathrm{Rh}$ films on $\mathrm{MgO}(001)$ remain magnetic, with moments of $1.95 \mu_{\mathrm{B}}$ and $1.21 \mu_{\mathrm{B}}$, respectively. However, Nokbin et al. [50] recently examined the $\mathrm{Rh} / \mathrm{MgO}$ system using a planewave basis and the projector augmented wave (PAW) method and found finite moments only for adsorption at the metastable $\mathrm{Mg}$ and "hollow" sites (see later); at the more stable $\mathrm{O}$ site the adsorbed ML was found to be non-magnetic, contradicting the results of $\mathrm{Wu}$ and Freeman.

In the present paper we will resolve the discrepancy between these two theoretical predictions. We will examine how magnetism in the $\mathrm{Rh} / \mathrm{MgO}(001)$ system is switched on and off, and how this depends upon the chemical bonding both within the adsorbed layer (the $\mathrm{Rh}-\mathrm{Rh}$ interactions) and between the ad-layer and the substrate (Rh-surface interactions). In so doing we hope to give a better guide to experimentalists looking for magnetism in $4 \mathrm{~d}$ transition metal systems, as well as a clearer understanding of the relationship between magnetism and bonding in metal on metal oxide systems in general. We will do this by relating the evolution of the magnetic moment to details of the electronic structure as a function of the adlayer-substrate distance and by discussing how this relates to the so-called Stoner criterion. The electronic structure of the $\mathrm{Rh} / \mathrm{MgO}$ system itself is also interesting in other chemical and technological contexts, most 
notably in heterogeneous catalysis. The functionality of $\mathrm{Rh} / \mathrm{MgO}$ catalysts has therefore been studied intensively experimentally; see for example Ref. [51,52].

Magnetic transitions are usually characterized and discussed in terms of the Stoner Criterion. $[53,54]$ This states that a system may have a non-zero magnetic moment if the exchange energy gained by becoming magnetic exceeds the kinetic energy penalty: hence if $I \cdot N\left(\varepsilon_{\mathrm{F}}\right)>1$, where $I$ is the Stoner parameter (see below), and $N\left(\varepsilon_{\mathrm{F}}\right)$ is the density of states (DOS) at the Fermi level, $\varepsilon_{\mathrm{F}}$. The density of states value $N\left(\varepsilon_{\mathrm{F}}\right)$ is inversely proportional to the bandwidth, and hence to the kinetic energy. The exchange energy is quantified by the Stoner parameter, $I$, which becomes large when the spatial confinement of the electrons at $\varepsilon_{\mathrm{F}}$ is large. Magnetism is thus favoured by small bandwidths (large $N\left(\varepsilon_{\mathrm{F}}\right)$ ) and by strong spatial confinement of the electronic wavefunctions (large $I$ ). The dependence of these on changes in the bonding are easy to track via plots of the DOS and of the charge and spin densities and derivatives of them. In principle, $I$ can be evaluated from the calculated wavefunction, but here we only need a framework for discussion, so we will consider only rough changes in $I$ as evidenced by changes in charge density and related properties. The more important contributions come from $N\left(\varepsilon_{\mathrm{F}}\right)$, however, since $I$ is dominated by the atomic region rather than its surroundings.

The alternative way to view the Stoner criterion is to say that magnetism is favoured when the states near $\varepsilon_{\mathrm{F}}$ (in this case the $4 \mathrm{~d}$ states of the $\mathrm{Rh}$ ) become more atomic-like. The extreme cases for $\mathrm{Rh}$ are thus the free atom (very sharp peaks in the DOS) and the bulk (fairly flat DOS at $\varepsilon_{\mathrm{F}}$ ). An important distinction should be drawn here, between atomic-like (i.e. localized) and "itinerant" (delocalized) magnetism. In the former case, the magnetic electrons are completely localized in atomic-like states, flat in momentum space, and do not contribute to electrical and thermal transport. In this case, only integer multiples of the single electron magnetic moment $\left(1 \mu_{B}\right)$ can occur. In the latter case, the magnetized electrons, while still fairly localized, belong none-the-less to a band with significant bandwidth. They are thus at least partially delocalized and do contribute to the transport properties. In this case only the total magnetic moment for the whole sample is an integer, so the moment per atom or per primitive unit cell may be any rational number from zero up to the free atom moment. In the present study we will find a crossover between the two regimes, as well as crossovers between magnetic and non-magnetic regimes.

\section{Method and system geometry}

All calculations were performed using periodic planewave density functional theory (DFT) with the Vienna Ab initio Simulation Package, VASP $[55,56]$. The PAW method $[57,58]$ was used, as implemented in VASP, with exchange and correlation described using the "PW91" generalized 
gradient approximation (GGA) [59]. Spin-polarized calculations were performed with a planewave cut-off energy of $500 \mathrm{eV}$ in all cases. (For comments on convergence, see below.) The valence electronic configurations of the atomic species employed were $\operatorname{Rh}\left(4 p^{6}, 5 s^{1}, 4 d^{8}\right)$, $\operatorname{Mg}\left(2 p^{6}, 3 s^{2}\right)$ and $\mathrm{O}\left(2 s^{2}, 2 p^{4}\right)$. Integration over k-points was done using Gaussian smearing with a Gaussian width equal to $0.1 \mathrm{eV}$ (except where otherwise noted) and the Brillouin zone was sampled over 6x6x6 and 6x6x1 Monkhorst-Pack (MP) [60] meshes for bulk and surface calculations, respectively. The structures were optimized until all the forces on unconstrained atoms were less than $0.01 \mathrm{eV} / \AA$. In this paper, we are concerned mainly with the magnetic properties of the $\mathrm{Rh} / \mathrm{MgO}(001)$ system. We report magnetic moments defined by

$$
\mu=\int_{-\infty}^{\varepsilon}\left[N_{\uparrow}(\varepsilon)-N_{\downarrow}(\varepsilon)\right] d \varepsilon
$$

where $N_{\uparrow}(\varepsilon)$ and $N_{\downarrow}(\varepsilon)$ are the majority and minority spin components, sometimes referred to as the $\alpha$ and $\beta$ components, respectively. The moments are thus given for the complete supercell, although we will also report projected moments on individual atoms. The atom projected moments were obtained using Wigner-Seitz projection [62] onto spheres of radii of 1.402, 1.058 and $0.820 \AA$ around the $\mathrm{Rh}, \mathrm{Mg}$ and $\mathrm{O}$ atoms, respectively.

Three different coverages (1/8, 1/2 and $1 \mathrm{ML}$ ) at three different sites (on top of $\mathrm{O}, \mathrm{Mg}$ and the hollow) were explored for the $\mathrm{Rh} / \mathrm{MgO}(001)$ system, as shown in Fig 1. The periodic models used were as follows: A single $\mathrm{Rh}$ layer was placed on one side of the $\mathrm{MgO}$ oxide slab and a vacuum gap of $15 \AA$ was used (see below.) In addition, calculations for isolated Rh layers and for $\mathrm{Rh}$ bulk and the $\mathrm{Rh}$ atom were performed (see below).

For $1 / 2$ and $1 \mathrm{ML}$ coverages, the calculation cell consisted of $8 \mathrm{MgO}$ layers plus a vacuum gap along $z$ and a $1 \mathrm{x} 1$ crystallographic unit cell in the $x$ and $y$ directions and. For the lowest coverage (1/8 ML), a 2x2 crystallographic unit cell in the $x$ and $y$ directions was used, with $4 \mathrm{MgO}$ layers along $z$. For all systems, all atoms were allowed to fully relax in all directions under the condition that the orthogonal unit cell was kept fixed at the optimized GGA bulk lattice parameter of $4.25 \AA$ in the $x$ and $y$ directions. (The experimental cell parameter of bulk $\mathrm{MgO}$ is $4.21 \AA$. Incidentally, our optimized bulk cell parameter for fcc bulk $\mathrm{Rh}$ is $3.85 \AA$ and the experimental value is $3.80 \AA[61])$.

To ensure that the planewave cutoff and k-point sampling had been properly converged for the various bulk, surface and adatom systems, several test calculations were performed. We found that increasing the k-point grids from $6 \times 6 \times 1 / 6 \times 6 \times 6$ to $8 \times 8 \times 1 / 8 \times 8 \times 8$ for the surface/bulk calculations altered the calculated total energies by $\mathrm{O}(0.01) \mathrm{eV} / \mathrm{O}(0.00001) \mathrm{eV}$. This was tested for clean bulk $\mathrm{MgO}$, and for the $\mathrm{Rh} / \mathrm{MgO}$ system with 1 and $1 / 2 \mathrm{ML} \mathrm{Rh}$ at the $\mathrm{O}$ site.) The calculated magnetic moments remained the same to $\mathrm{O}(0.01) \mu_{\mathrm{B}}$. Increasing the planewave cutoff 
from $500 \mathrm{eV}$ to $600 \mathrm{eV}$ resulting in changes of $\mathrm{O}(0.0001) \mathrm{eV}$ in the total energy of the free atom but for all other cases it made changes of $\mathrm{O}(0.1) \mathrm{eV}$ in the total energy. This is large, but we are only interested in differences between total energies, and these are much better: the adsorption energies at the $\mathrm{O}$ and hollow sites with $1 \mathrm{ML}$ and $1 / 2 \mathrm{ML}$ coverage are converged to $\mathrm{O}(0.01) \mathrm{eV}$, while the energy differences between the $\mathrm{O}$ and hollow sites are converged to $\mathrm{O}(0.001) \mathrm{eV}$. The magnetic moments are all converged to $\mathrm{O}(0.001) \mu_{\mathrm{B}}$ or better with a $500 \mathrm{eV}$ planewave cutoff. Regarding the models themselves, increasing the number of $\mathrm{MgO}$ layers in the slab from 4 to 5 for $1 / 8 \mathrm{ML}$ altered the $\mathrm{Rh}$ adsorption energy by only $\mathrm{O}(0.001) \mathrm{eV}$ at the $\mathrm{O}$ site. Increasing the vacuum gap from 15 to $20 \AA$ alters the clean surface total energy by less than $0.001 \mathrm{eV}$, so adsorption energies etc should be still better converged. Indeed, reducing the vacuum gap to 10 $\AA$ only produces total energy errors of less than $0.01 \mathrm{eV}$ (relative to a $20 \AA$ gap), so results should be well converged even when the Rh atom is placed high above the surface.

\section{Results and discussion}

\subsection{Magnetic properties of the $\mathrm{Rh}$ atom and $\mathrm{Rh}$ isolated layers}

As reference systems, the isolated $\mathrm{Rh}$ atom and bulk $\mathrm{Rh}$ metal were studied, together with the three different isolated Rh MLs, corresponding to the three different coverages. These were all studied using the same potentials, plane-wave cutoff etc. as for the $\mathrm{Rh} / \mathrm{MgO}$ systems. The DOS for bulk and atomic $\mathrm{Rh}$ and for the three isolated MLs are shown in Fig 2, while the magnetic moments obtained for the isolated MLs are listed in Table 1.

For atomic $R h$, the expected magnetic moment is $3 \mu_{\mathrm{B}}$, since the ground state configuration is $4 d^{8} 5 s^{1} .2 \mu_{B}$ of the moment comes from the $4 d$ shell and $1 \mu_{B}$ from the $5 \mathrm{~s}$. Using the Gaussian width of $0.1 \mathrm{eV}$ for the smearing - to maintain consistency with all our other results - we find calculated magnetizations of $1.97 \mu_{\mathrm{B}}$ and $1.96 \mu_{\mathrm{B}}$ in supercells of 10.0 and $20.0 \AA$, respectively. (This becomes $1.39 \mu_{\mathrm{B}}$ using the Wigner-Seitz projection, in agreement with the previous value of $1.38 \mu_{\mathrm{B}}$ from Moruzzi and Marcus [63].) The problem arises partly because the PAW potential used in this study has been generated to obtain accurate results for Rh in compounds and molecules, not for the free atom, so the smearing of $0.1 \mathrm{eV}$ is sufficient to mix the $4 \mathrm{~d}$ and $5 \mathrm{~s}$ giving us an actual configuration of $4 \mathrm{~d}^{8.5} 5 \mathrm{~s}^{0.5}$, instead of that expected. This gives contributions of $1.5 \mu_{\mathrm{B}}$ and $0.5 \mu_{\mathrm{B}}$ from the $4 \mathrm{~d}$ and $5 \mathrm{~s}$ shells, respectively - hence the $2 \mu_{\mathrm{B}}$ total obtained. In principle this can be corrected by fixing the occupancies of the Kohn-Sham orbitals to force the expected $4 d^{8} 5 s^{1}$ configuration. However, the energies obtained by this method are not very meaningful. Instead we can overcome the problem by reducing the smearing to $0.001 \mathrm{eV}$. This gives us the correct $4 d^{8} 5 \mathrm{~s}^{1}$ configuration, a magnetization of $3 \mu_{\mathrm{B}}$ and a total energy $0.222 \mathrm{eV}$ 
lower. However, for the purpose of consistency, we have used the Rh atom results calculated with a Gaussian width of $0.1 \mathrm{eV}$ for the calculation of the adsorption energies (see later).

Bulk $R h$ is non-magnetic. The $\mathrm{Rh}-\mathrm{Rh}$ bonding will, of course, make the $4 \mathrm{~d}$ electrons less localized, reducing $I$. As seen in $\mathbf{F i g} \mathbf{2}$, it also leads to a broad $4 \mathrm{~d}$ band and hence to a low $N\left(\varepsilon_{\mathrm{F}}\right)$. The Stoner criterion is thus not met and so there is no splitting between $N_{\uparrow}(\varepsilon)$ and $N_{\downarrow}(\varepsilon)$.

The isolated free $R h$ monolayers all consist of 2D square lattices with nearest-neighbour $\mathrm{Rh}-\mathrm{Rh}$ distances of $8.50,4.25$ and $3.00 \AA$, corresponding to the $1 / 8,1 / 2$ and 1 ML systems, respectively. Calculations were done in supercells of the same dimensions as those used for the full $\mathrm{Rh} / \mathrm{MgO}$ slab cases. As we go from $1 / 8$ to $1 / 2$ to $1 \mathrm{ML}$, the $4 \mathrm{~d}$ band progressively widens as the nearest neighbour distance decreases, resulting in increased $\mathrm{Rh}-\mathrm{Rh}$ interaction, and specifically d-d hybridization, within the layer. $N\left(\varepsilon_{\mathrm{F}}\right)$ thus decreases in the order $1 / 8 \mathrm{ML}>1 / 2 \mathrm{ML}>1 \mathrm{ML}$, with the first value closely resembling the free atom and the last resembling bulk $\mathrm{Rh}$. The Stoner criterion is clearly fulfilled for all of the isolated MLs, but more strongly for the 1/8 ML layer and less strongly for the 1 ML layer, as evidenced by the calculated total magnetic moments of $1.97,1.69$ and $1.57 \mu_{\mathrm{B}}$ /atom for the $1 / 8,1 / 2$ and $1 \mathrm{ML}$, respectively. (The latter figure is in good agreement with the previous calculations of Zhu et al.[25]) If the Wigner-Seitz projections described earlier are used this becomes $1.40,1.40$ and $1.46 \mu_{\mathrm{B}} /$ atom.

\subsection{Magnetic properties of the $\mathrm{Rh} / \mathrm{MgO}(001)$ interface}

Table 1 also lists the total magnetic moments of the whole cell for the various $\mathrm{Rh} / \mathrm{MgO}$ systems.

Table 2 lists the corresponding Wigner-Seitz-projected moments for the Rh atoms and for those surface-layer $\mathrm{Mg}$ and $\mathrm{O}$ atoms which lie closest to the adsorbed $\mathrm{Rh}$ atoms. The moments are shown at the optimal Rh-surface distances for the various cases, as listed. The main contribution to the magnetic moment is always from $\mathrm{Rh}$, with small induced moments in the $\mathrm{O}$ atoms in the surface layer. The contribution of the Mg atoms is negligible. From our previous work [43] and [50] we know that the most stable adsorption site is the $\mathrm{O}$ site for all coverages, with the hollow and $\mathrm{Mg}$ sites being only metastable. For the $1 \mathrm{ML}$ cases, the $\mathrm{O}, \mathrm{Mg}$ and hollow sites have total magnetic moments (for the whole cell) of $0.00,1.48$ and $1.42 \mu_{B} / \mathrm{Rh}$ atom, respectively. For the $1 / 2 \mathrm{ML}$, all the systems are magnetic with moments of $1.00,1.58$ and $1.13 \mu_{\mathrm{B}} / \mathrm{Rh}$ atom at the $\mathrm{O}$, $\mathrm{Mg}$ and hollow adsorption sites, respectively. The magnetic moment for $1 / 8 \mathrm{ML}$ at the $\mathrm{O}$ site is $1.00 \mu_{B} / \mathrm{Rh}$ atom. Hence the adsorption site dependence of the magnetic moments comes in the order $\mathrm{O}<$ hollow $<\mathrm{Mg}$, independent of coverage. The magnetic moments at the $\mathrm{O}$ site increase in the order $1 \mathrm{ML}<1 / 2 \mathrm{ML}<1 / 8 \mathrm{ML}$. Qualitatively, the coverage (or Rh density) dependence of the magnetism in the adsorbed MLs is thus similar to that of isolated free Rh MLs, but it may be more informative to note that the magnetic moments have the opposite trend to the ML- 
substrate interaction energies: the larger the interaction energy, the smaller the magnetic moment. This agrees well with findings in the literature (see, for example, Ref. [64]).

The only $\mathrm{Rh} / \mathrm{MgO}$ case for which we do not get a magnetic moment is for $1 \mathrm{ML}$ at the $\mathrm{O}$ site. This is confirmed by the plot in Fig 3, which shows the energy as a function of total magnetic moment, taken from a series of Fixed Spin Moment (FSM) calculations, [65,66] (Each calculation is performed by minimizing the total energy subject to the constraint that the total magnetic moment in the supercell is equal to a pre-determined values.) The curve is fairly linear, with a slope of approximately $0.05 \mathrm{eV} / \mu_{\mathrm{B}}$, and a ground state of $\leq 0.1 \mu_{\mathrm{B}}$. (The figure does indicate that the energy is very flat for moments $\leq 0.2 \mu_{\mathrm{B}}$, but we believe this to be a numerical problem). The zero moment for $1 \mathrm{ML}$ at the $\mathrm{O}$ site directly contradicts the results of $\mathrm{Wu}$ et al. [42], who found a magnetic moment of $1.21 \mu_{\mathrm{B}}$. To see why, we show in Fig 4 the magnetic moment as a function of Rh-surface distance $d$. The curves for the $\mathrm{O}$ site and for all the other sites and coverages were obtained by taking the relaxed atomic positions from the optimized $\mathrm{Rh} / \mathrm{MgO}$ structure for the case in question, and then keeping all the positions within both the $\mathrm{MgO}$ slab and the $\mathrm{Rh}$ layer fixed and just rigidly moving the $\mathrm{Rh}$ layer relative to the $\mathrm{MgO}$ surface with no further relaxation. For all of the sites, and at all coverages, the Rh layer starts out with magnetic moments the same as those of the isolated layers when $d \approx 5 \AA$. In most cases this starts to fall for $d \approx 2-3 \AA$, as the Rh layer starts to interact with the surface, before dropping to zero at some shorter distance. The details of the curves vary with adsorption site, indicating the sensitivity of the Rh magnetism to details of the environment around the adsorbed atom. They also vary strongly with coverage, indicating the sensitivity to the weakening of the intra-layer d$\mathrm{d}$ hybridization - in effect, sensitivity to the dimensionality. However, in almost all cases, the optimal distance $d_{o}$ occurs before the moment falls to zero. The exception is for $1 \mathrm{ML}$ at the $\mathrm{O}$ site, where $d_{o}=2.10 \AA$, with the moment having fallen to zero between 2.20 and $2.15 \AA$. Wu and Freeman [42] obtained an optimal distance of $d_{o}=2.34 \AA$. On our plot this would result in a moment of $\sim 1.4 \mu_{\mathrm{B}}$, which is rather close to the moment they obtained. They chose to fix the lattice constant in the $x$ and $y$ directions to the experimental value (rather than the relaxed GGA one), which leads to a small unphysical strain in the $x-y$ plane. This in turn will have adversely affected their relaxed bond lengths and angles by a small but possibly significant amount. Although it is not conclusive, we also find a more stable interface energy $(0.95 \mathrm{eV}$ versus 0.84 $\mathrm{eV})$.

\subsection{The relationship between bond formation and magnetism at the $\mathrm{Rh} / \mathrm{MgO}(001)$ interface}

Figs 5 to 9 illustrate the changes in the electronic, magnetic and bonding behaviour for various cases as a function of $d$. More specifically, the DOS, the Rh atom projected DOS, as well as the charge density, $\rho$, the charge density difference $\Delta \rho$ and the spin density $\sigma=\rho_{\uparrow}+\rho_{\downarrow}$, are plotted 
as a function of $d$. The $\Delta \rho$ shown is the charge density $\rho$ for the complete $\mathrm{Rh} / \mathrm{MgO}$ system minus the sum of the charge densities for the isolated $\mathrm{Rh}$ layer (i.e. without the $\mathrm{MgO}$ slab) and the clean $\mathrm{MgO}$ slab (i.e. without the $\mathrm{Rh}$ layer), using the same atomic positions in the three calculations involved. In other words it shows the changes in $\rho$ resulting from the interaction between the layer and the slab. It provides a neat way to view the real-space changes in the electronic structure resulting from the interactions and bonding between the systems.

\subsection{ML Rh at the O site}

Fig 5 shows the behaviour of the $1 \mathrm{ML} \mathrm{Rh/MgO}$ over the $\mathrm{O}$ site. For large $d(\geq 3.00 \AA$ ), the total DOS has two distinct peaks (although the Rh-projected DOS indicates more substructure). The strongest peak lies just below $\varepsilon_{F}$, leading to a large value for $N\left(\varepsilon_{\mathrm{F}}\right)$. The $\Delta \rho$ maps show the electron redistribution in the $\mathrm{Rh}$ layer caused by the interaction with the oxide substrate and the redistribution is seen to be modest for largest $d$ value (rightmost figure in the third row of $\mathbf{F i g} \mathbf{5}$ ), suggesting that the Stoner parameter $I$ is also large. We therefore expect $I \bullet N\left(\varepsilon_{\mathrm{F}}\right)$ to be large, meeting the Stoner criterion: indeed we find a reasonably strong splitting between the spin up $\left(N_{\uparrow}\right)$ and down $\left(N_{\downarrow}\right)$ components of the Rh $4 \mathrm{~d}$ levels and a finite magnetic moment of $1.55 \mu_{\mathrm{B}}$, similar to that of the isolated Rh layer.

As the Rh layer is brought in from $4.78 \AA$ to $2.25 \AA$ above the surface, the electronic structure undergoes a qualitative change as the layer starts to interact with the surface, as seen in the DOS. At the same time the charge density in the oxide surface layer is rearranged, but seemingly in a gradual fashion, as evidenced by the $\Delta \rho$ maps. The $\sigma$ maps show that a magnetic moment is induced on the surface oxygen atoms as $d$ is decreased from 3.78 to $3.00 \AA$, leading to a slight rise in the magnetization, in agreement with Fig 4. The electron density is seen to decrease between the $\mathrm{Rh}$ and the surface $\mathrm{O}$ (see the $\Delta \rho$ maps), as well as in the region above the $\mathrm{Rh}$ atom, and is spread out in between the $\mathrm{Rh}$, in the region above the surface $\mathrm{Mg}$. Hence it appears that the "in-layer" Rh-Rh interaction (d-d hybridization) is actually enhanced by the Rh-surface interaction. This results in the $\mathrm{Rh} 4 \mathrm{~d}$ electrons becoming gradually less localized (and hence the Stoner parameter $I$ gradually smaller) as $d$ is reduced. The $4 \mathrm{~d}$ band in the DOS becomes broader, and the main peaks become weaker and move to lower energies, resulting in a much lower value of $N\left(\varepsilon_{\mathrm{F}}\right)$.

However, what it at remarkable - at least at first glance - is that the qualitative changes in the electronic structure, as evidenced by the change in the Rh-projected DOS at $d=3.00 \Rightarrow 2.25 \AA$, leave the $\mathrm{Rh}$ magnetization virtually unchanged. Instead, when the moment does disappear, it does so sharply at $d=2.25 \Rightarrow 2.10 \AA$, even though there is almost no visible change in the DOS, $\rho$ or $\Delta \rho$ here. In other words, it is not in itself the adhesion of the $\mathrm{Rh}$ to the surface that switches 
off the magnetism. Certainly, the qualitative changes to the electronic structure at longer distances $d o$ reduce $I \bullet N\left(\varepsilon_{\mathrm{F}}\right)$, and but they do not take it below 1 . Instead it is a gradual transition, in which the value of $I \bullet N\left(\varepsilon_{\mathrm{F}}\right)$ falls smoothly, finally dipping below 1 without any qualitative changes to the electronic structure taking place at all. In fact, this results in the magnetization vs $d$ curve shown for the $\mathrm{O}$ site in figure $4 \mathrm{a}$ ) being very close to the idealized Stoner criterion plots found in textbooks, with our $d$ playing the role of (and presumably proportional to) $I \bullet N\left(\varepsilon_{\mathrm{F}}\right)$.

\subsection{2 $1 / 2$ and $1 / 8$ ML Rh at the $O$ site}

Figures 6 and 7 show the DOS, projected DOS, $\Delta \rho$ and $\sigma$ as a function of $d$ for the cases of, respectively, $1 / 2$ and $1 / 8 \mathrm{ML}$ Rh over O. The total DOS, $\rho, \Delta \rho$ and $\sigma$ are very similar to the 1 ML systems although the features are smaller due to the lower density of $\mathrm{Rh}$. The $\mathrm{Rh}$ atoms themselves are further apart, so the Rh-Rh interactions are much smaller, leading to both higher localization (larger $I$ ) and narrower Rh projected DOS (larger $N\left(\varepsilon_{\mathrm{F}}\right)$ ), and hence to stronger magnetic effects. Indeed, the projected DOS for the $1 / 8 \mathrm{ML}$ case is very atomic-like, and the resulting magnetization should probably be an integer multiple of $\mu_{B}$ for all $d$. It is for $d<3 \AA$, but, unfortunately, we have convergence problems above this, as seen in Fig $4 \mathrm{~b}$. These are no doubt due to the same problem met with the free atom. We could in principle get around them by forcing the expected Kohn-Sham occupancies, but the results would not be very informative certainly not if we wanted to know where the $2 \mu_{\mathrm{B}}$ to $3 \mu_{\mathrm{B}}$ transition occurs. Worse, here we cannot get around the problems by simply relying on a smaller smearing, as that would also make the description of the slab worse. Instead, we have examined the results of a range of different k-point integration methods. (The k-point grid itself is still well converged using $6 \times 6 \times 1^{\ddagger}$.) We have tried both Gaussian and Methfessel-Paxton (order 1 and 2) [67] smearing with widths of 0.1 and $0.005 \mathrm{eV}$, as well as Fermi smearing and the tetrahedron method with and without Blöchl corrections [68]. The values plotted in Fig. 4 use Gaussian smearing with a width of $0.1 \mathrm{eV}$, while the error bars indicate the highest and lowest values obtained with the other schemes: they essentially vary from 2 to $3 \mu_{\mathrm{B}}$ depending upon whether the $\mathrm{Rh}$ layer or the $\mathrm{MgO}$ slab is badly treated. Nevertheless, the results are mostly consistent with an integer value of $2 \mu_{\mathrm{B}}$, which is itself consistent with the values obtained for the isolated layer and for the free Rh atom.

As a function of increasing Rh density (hence, increasing d-d hybridization) we thus see a crossover between itinerant and localized (atomic) magnetism. The 1/8 ML moment itself changes from 2 (or perhaps 3 ) $\mu_{B}$ to $1 \mu_{B}$ at about $d=2.8 \AA$, accompanied this time by changes in $\Delta \rho$. We cannot find the change from 1 to $0 \mu_{B}$, as it happens at such short distances that the results are difficult to converge numerically and not very meaningful physically. The results for

\footnotetext{
* For example, for $d=5 \AA$ the energy difference obtained between fixed magnetizations of 2 and $3 \mu_{\mathrm{B}}$ is 0.0927 for $6 \times 6 \times 1,0.0911 \mathrm{eV}$ for $8 \times 8 \times 1$ and $0.0910 \mathrm{eV}$ for $10 \times 10 \times 1$, using Gaussian smearing with width $=0.005 \mathrm{eV}$.
} 
the very small $d$ values, given here and elsewhere, are reported to help examine the magnetic transitions in the $\mathrm{Rh} / \mathrm{MgO}$ system, but the accuracy and physicality of these results are certainly much reduced compared to the results for, say, $d>1.5 \AA$.

For the $1 / 2 \mathrm{ML}$ case, there is again a qualitative change in the charge density and bonding accompanying the magnetic transition. The DOS and projected DOS show distinct changes a little above the magnetic transition (around $d \approx 1.5 \AA$ ) and a gradual decrease in $N\left(\varepsilon_{\mathrm{F}}\right)$ at the transition itself. Meanwhile, $\Delta \rho$ shows the charge being pushed outwards to accumulate above the Rh atoms $(d=0.75 \AA)$ in an area which shows charge depletion for larger $d$ values. Hence the magnetic transition seems to be driven in this case by a gradual change in $N\left(\varepsilon_{\mathrm{F}}\right)$ and a sudden change in $I$ caused by the Rh crashing into the $\mathrm{O}$ atom below it. Meanwhile, the crossover from itinerant to localized magnetism is actually seen here as a function of $d$. Around the optimal distance $\left(d_{o}=2.10\right)$ and below we find atomic-like DOS and integer moments. For larger distances we find non-integer moments. The accompanying changes in $\rho, \Delta \rho$ and $\sigma$ are again gradual, so, like the 1ML magnetic transition, the itinerant/localized magnetism crossover is not directly linked to bonding changes.

\subsubsection{1/2 and 1 ML Rh at the Mg and Hollow sites}

The total and projected DOS, $\Delta \rho$ and $\sigma$ for $1 \mathrm{ML}$ at the two metastable sites are shown in Figs 8 and 9. The total and projected DOS and $\sigma$ are actually strikingly similar to the DOS for $1 \mathrm{ML} \mathrm{Rh}$ at the $\mathrm{O}$ site (above the transition), and show a similar evolution with $d$. The $\mathrm{Rh} 4 \mathrm{~d}$ electrons become gradually more spread out and the DOS broadens slightly, weakening the magnetic moment. The $\Delta \rho$, on the other hand is very different. Comparing $\Delta \rho$ for all three sites as $d$ is reduced it always shows a depletion of charge between $\mathrm{Rh}$ and $\mathrm{O}$, and an accumulation of charge between $\mathrm{Rh}$ and $\mathrm{Mg}$. For the $\mathrm{O}$ site, this leads to accumulation between the $\mathrm{Rh}$ atoms and depletion above and below. For the Mg site, it leads to depletion between the Rh atoms and accumulation below and above. For the hollow site it leads to a twisted, diagonal pattern, with $\mathrm{Rh}$ atoms separated by regions of both accumulation and depletion. Hence, the d-d hybridization is enhanced for the $\mathrm{O}$ site (decreasing both $N\left(\varepsilon_{\mathrm{F}}\right)$ and $I$ ), but is reduced at the other two. This appears to be the reason why $\mathrm{Rh}$ is non-magnetic at $d_{0}$ at the $\mathrm{O}$ site, but remains magnetic at the other two.

For both sites, as $d$ is reduced below $2 \AA$ the $\mathrm{Rh}$ and $\mathrm{Mg}$ atoms start to overlap more strongly, an induced moment appears for a while on the neighbouring $\mathrm{Mg}$ atoms. This leads to the increases in magnetization at $d \approx 1.5 \AA$ and $1.25 \AA$ for the hollow and Mg sites, respectively, as seen in Fig 4a. Finally, for the hollow site only weak changes are seen at the magnetic transition, (mostly in $\Delta \rho$ for $\mathrm{d} \geq 2.5 \AA$ ) but for the $\mathrm{Mg}$ site a very distinct change is observed in $\Delta \rho$ and DOS. Electron density is suddenly transferred from the $\mathrm{Rh}$ and neighbouring $\mathrm{Mg}$ atoms to $\mathrm{Mg}$ 
and $\mathrm{O}$ atoms in the second layer of the $\mathrm{MgO}$ slab, and this sudden reduction in the localization kills off the magnetic moment.

\section{Conclusion}

We previously $[43,50]$ showed that the most stable site for $\mathrm{Rh}$ adsorption on $\mathrm{MgO}(001)$ is the $\mathrm{O}$ site and found that it is nonmagnetic at $1 \mathrm{ML}$ coverage, in contradiction to an earlier study. Here we have confirmed that it is indeed non-magnetic at $1 \mathrm{ML}$ coverage, but that it is magnetic at $1 / 2$ and 1/8 ML coverages, and at the metastable $\mathrm{Mg}$ and hollow sites. The energy difference between magnetic and non-magnetic ground states is $0.1 \mathrm{eV}$ for $1 / 2 \mathrm{ML}$ at the $\mathrm{O}$ site; the others are similar. We have then studied the dependence of the Rh magnetic moment upon the chemical interactions within the layer and between the layer and the surface by examining the DOS, projected DOS, $\rho, \Delta \rho$ and $\sigma$ as a function of coverage, site and surface-adlayer distance $d$.

As the $\mathrm{Rh}$ layer is brought towards the $\mathrm{MgO}$ slab, they start to interact. At some point the shape of the DOS, particularly the Rh-projected DOS, changes qualitatively, illustrating changes in the $\mathrm{Rh}-\mathrm{Rh}$ and Rh-surface interactions. $\Delta \rho$ is seen to change more gradually as a function of $d$; thus some bonding changes appear to be more easily detected in the DOS curves. As $d$ shrinks, the Rh projected DOS gets gradually wider and $N\left(\varepsilon_{\mathrm{F}}\right)$ gradually smaller. For Rh adsorbed at the $\mathrm{O}$ site, the charge becomes more spread out into the region between the Rh atoms, thus becoming less localized, so that the Stoner parameter $I$ shrinks. At some point the Stoner criterion ceases to be fulfilled and the magnetism switches off. For the 1/2 and 1/8 ML O site cases, as well as for the $1 \mathrm{ML} \mathrm{Mg}$ site case, this happens at the same point as the qualitative changes in the DOS, and hence in the bonding. For the $1 \mathrm{ML} \mathrm{O}$ and hollow site cases, on the other hand, it does not. In other words, the presence of a magnetic moment and the formation or enhancement of the intralayer or layer-slab bonding are not directly connected.

In addition to the magnetic transition and its variations with coverage and site, we have also observed a crossover from localized (integer moment) to delocalized (fractional moment) magnetism as a function of coverage. The lower the coverage the smaller the d-d hybridization, and hence the more localized the magnetism.

As a function of $\mathrm{Rh}-\mathrm{Rh}$ and $\mathrm{Rh}$-surface interaction strengths we thus find regions of both localized and itinerant magnetism as well as non-magnetic regions. This is illustrated in Fig $\mathbf{1 0}$, which shows the magnetic moment plotted against estimates of the $\mathrm{Rh}-\mathrm{Rh}$ and $\mathrm{Rh}$-surface interaction strengths. The $\mathrm{Rh}-\mathrm{Rh}$ interaction is estimated by the formation energies of the isolated layers and the Rh-surface interaction strength by the adsorption energies. Moments are plotted for the isolated layers and for all three adsorption sites, at all coverages calculated, but only at the optimal distances. The trend is that increasing either the Rh-Rh interaction (d-d 
hybridization) or the Rh-surface interaction leads to reduced magnetic moments, and increasing the d-d hybridization drives the system from localized to itinerant magnetism. Having studied here only one $4 \mathrm{~d}$ metal on one surface it is difficult to know whether the phase diagram shown in Fig 10 is generic, but the present example at least suggests a strategy. When searching for magnetic $4 \mathrm{~d}$ and $5 \mathrm{~d}$ thin films and monolayers one might want to examine systems in which the adlayer and the surface interact only weakly and which preferably have low coverage in order to keep the average d-d hybridization low. In the case of $\mathrm{Rh}$ on the $\mathrm{MgO}$ (100) surface it is certainly necessary to go to coverages below 1 ML. The critical coverage lies between 1/2 and 1 ML, but where exactly we cannot say; this is perhaps an important question for future computational work.

Further computational work is also needed to determine how important the structural ordering of the $\mathrm{Rh}$ adlayer is for the presence of magnetism at low coverages. We are not aware of any experiments preparing or characterizing submonolayer films of $\mathrm{Rh}$ on $\mathrm{MgO}$, but island growth is preferred for several other metal/MgO systems. [69,70,71] For a specific coverage the key to finding magnetism is having only weak $\mathrm{d}$-d hybridization, so island size is a potential issue. Large islands will probably be non-magnetic, while small islands or homogeneously distributed adatoms are likely to remain magnetic. These could probably be ordered or disordered, or even link to form networks, as long as the Rh-Rh coordination remains low. One of these latter situations is the most promising for an experimental demonstration of magnetism in $\mathrm{Rh} / \mathrm{MgO}$ systems. For instance, recent calculations [72] have predicted that growth of a disordered but homogeneous film is favoured over island growth in the presence of surface defects, a situation which can probably be engineered during substrate preparation. Low temperature experiments are likely to be needed in order to stabilize both structure and magnetism.

\section{Acknowledgements}

This work was supported by the Swedish Foundation for International Cooperation in Research and Higher Education, (STINT) and the Swedish Research Council (VR). S.N. wishes to thank the Thailand Research Fund (TRF) for a Royal Golden Jubilee Ph.D. fellowship (3.C.KU/43/A.2). The authors also acknowledge the use of the Ngorongoro computer system at the Department of Information Technology, Uppsala University and the Lenngren and Swegrid facilities run by the Swedish National Infrastructure for Computing. We would also like to thank Susanne Mirbt for helpful discussions. 


\section{References}

[1] A. J. Cox, J. G. Louderback, L. A. Bloomfield, Phys. Rev. Lett. 71 (1993) 923.

[2] A. J. Cox, J. G. Louderback, S. E. Apsel, L. A. Bloomfield, Phys. Rev. B 49 (1994) 12295.

[3] C. Barreteau, R. Guirado-Lopez, D. Spanjaard, M. C. Desjonqueres, A. M. Oles, Phys. Rev. B 61 (2000) 7781.

[4] Z.-Q. Li, J.-Z. Yu, K. Ohno, Y. Kawazoe, Sci. Rep. Res. Inst. Tohoku Univ. Phys. Chem. Metall. 40 (1995) 297.

[5] J. Yang, K. Deng, C. xiao, K. Wang, Phys. Status Solidi B 195 (1996) 549.

[6] B. V. Reddy, S. N. Khanna, B. I. Dunlap, Phys. Rev. Lett. 70 (1993) 3323.

[7] K. Lee, Z. Phys. D: At., Mol. Clusters 40 (1997) 164.

[8] Y. Jinlong, F. Toigo, W. Kelin, Surf. Rev. Lett. 3 (1996) 323.

[9] V. Kumar, Y. Kawazoe, Eur. Phys. J. D 24 (2003) 81.

[10] B. V. Reddy, S. K. Nayak, S. N. Khanna, B. K. Rao, P. Jena, Phys. Rev. B 59 (1999) 5214.

[11] K. Wildberger, V. S. Stepanyuk, P. Lang, R. Zeller, P. H. Dederichs, Phys. Rev. Lett. 75 (1995) 509.

[12] D. I. Bazhanov, W. Hergert, V. S. Stepanyuk, A. A. Katsnelson, P. Rennert, K. Kokko, C. Demangeat, Phys. Rev. B 62 (2000) 6415.

[13] S. Blügel, Phys. Rev. Lett. 68 (1992) 851.

[14] S. C. Wu, K. Garrison, A. M. Begley, F. Jona, P. D. Johnson, Phys. Rev. B 49 (1994) 14081.

[15] J.-H. Cho, M. Scheffler, Phys. Rev. Lett. 78 (1997) 1299.

[16] L. M. Garcia-Cruz, R. Baquero, Rev. Mex. Fis. 49 (2003) 317.

[17] J. Redinger, S. Bluegel, R. Podloucky, Phys. Rev. B 51 (1995) 13852.

[18] I. Turek, J. Kudrnovsky, M. Sob, V. Drchal, P. Weinberger, Phys. Rev. Lett. 74 (1995) 2551.

[19] A. M. N. Niklasson, S. Mirbt, H. L. Skriver, B. Johansson, Phys. Rev. B 56 (1997) 3276.

[20] R. Wu, A. J. Freeman, Phys. Rev. B 45 (1992) 7222.

[21] S. Blügel, Solid State Commun. 84 (1992) 621.

[22] O. Eriksson, R. C. Albers, A. M. Boring, Phys. Rev. Lett. 66 (1991) 1350.

[23] B. Ujfalussy, L. Szunyogh, P. Weinberger, Phys. Rev. B 51 (1995) 12836. 
[24] A. Mokrani, H. Dreysse, S. Bouarab, C. Demangeat, J. Magn. Magn. Mater. 113 (1992) 201.

[25] M. J. Zhu, D. M. Bylander, L. Kleinman, Phys. Rev. B 43 (1991) 4007.

[26] Z. V. Sljivancanin, F. R. Vukajlovic, J. Phys.: Condens. Matter 10 (1998) 8679.

[27] C. S. Chang, L. H. Cho, J. I. Lee, S. C. Hong, R. Wu, A. J. Freeman, J. Magn. Magn. Mater. 177-181 (1998) 1255.

[28] A. E. Garcia, V. Gonzalez-Robles, R. Baquero, Phys. Rev. B 59 (1999) 9392.

[29] A. B. Hayden, T. Valla, D. P. Woodruff, J. Phys.: Condens. Matter 7 (1995) 9475.

[30] S. Blügel, Phys. Rev. B 51 (1995) 2025.

[31] I. Chado, F. Scheurer, J. P. Bucher, Phys. Rev. B 64 (2001) 094410/1.

[32] G. A. Mulhollan, R. L. Fink, J. L. Erskine, Phys. Rev. B 44 (1991) 2393.

[33] C. Liu, S. D. Bader, Phys. Rev. B 44 (1991) 12062.

[34] C. Demangeat, S. Bouarab, H. Dreysse, Int. J. Mod. Phys. B 7 (1993) 488.

[35] W. Hergert, P. Rennert, C. Demangeat, H. Dreysse, Surf. Rev. Lett. 2 (1995) 203.

[36] R. Pfandzelter, G. Steierl, C. Rau, Phys. Rev. Lett. 74 (1995) 3467.

[37] L. Chen, R. Wu, N. Kioussis, J. R. Blanco, J. Appl. Phys. 81 (1997) 4161.

[38] P. Kruger, A. Rakotomahevitra, G. Moraitis, J. C. Parlebas, C. Demangeat, Physica B 237-238 (1997) 278.

[39] P. Kruger, J. C. Parlebas, G. Moraitis, C. Demangeat, Computational Materials Science $10(1998) 265$.

[40] C. Li, A. J. Freeman, Phys. Rev. B 43 (1991) 780.

[41] Y. Y. Huang, C. Liu, G. P. Felcher, Phys. Rev. B 47 (1993) 183.

[42] R. Wu, A. J. Freeman, Phys. Rev. B 51 (1995) 5408.

[43] S. Nokbin, J. Limtrakul, K. Hermansson, Surf. Sci. 566-568 (2004) 977.

[44] C. Martinez Boubeta, C. Clavero, J. M. Garcia-Martin, G. Armelles, A. Cebollada, L. Balcells, J. L. Menendez, F. Peiro, A. Cornet, M. F. Toney, Phys. Rev. B 71 (2005) 014407/1.

[45] F. Didier, J. Jupille, Surf. Sci. 307-309 (1994) 587.

[46] G. Renaud, A. Barbier, O. Robach, Phys. Rev. B 60 (1999) 5872.

[47] O. Robach, G. Renaud, A. Barbier, Phys. Rev. B 60 (1999) 5858.

[48] M. P. Delplancke, P. Delcambe, L. Binst, M. Jardinier-Offergeld, F. Bouillon, Thin Solid Films 143 (1986) 43. 
[49] P. Blaha, K. Schwarz, P. Sorantin, S. B. Trickey, Comput. Phys. Commun. 59 (1990) 399.

[50] S. Nokbin, C.W.M. Castleton, J. Limtrakul, K. Hermansson, (to be published).

[51] F. Solymosi, J. Cserenyi, L. Ovari, J. Catal. 171 (1997) 476.

[52] H. Y. Wang, E. Ruckenstein, J. Catal. 186 (1999) 181.

[53] M. M. Sigalas, D. A. Papaconstantopoulos, Phys. Rev. B 50 (1994) 7255.

[54] G. Fabricius, A. M. Llois, H. Dreysse, Phys. Rev. B 48 (1993) 6665.

[55] G. Kresse, J. Hafner, Phys. Rev. B 47 (1993) 558.

[56] G. Kresse, J. Furthmueller, Phys. Rev. B 54 (1996) 11169.

[57] P. E. Blöchl, Phys. Rev. B 50 (1994) 17953.

[58] G. Kresse, D. Joubert, Phys. Rev. B 59 (1999) 1758.

[59] J. P. Perdew, J. A. Chevary, S. H. Vosko, K. A. Jackson, M. R. Pederson, D. J. Singh, C. Fiolhais, Phys. Rev. B 46 (1992) 6671.

[60] H. J. Monkhorst, J. D. Pack, Phys. Rev. B 13 (1976) 5188.

[61] H. P. Singh, Acta Crystallogr., Sect. A: Found. Crystallogr. 24 (1968) 469.

[62] A. Eichler, J. Hafner, J. Furthmueller, G. Kresse, Surf. Sci. 346 (1996) 300.

[63] V. L. Moruzzi, P. M. Marcus, Phys. Rev. B 39 (1989) 471.

[64] A. J. Freeman, R. Q. Wu, J. Magn. Magn. Mater. 100 (1991) 497.

[65] A.R. Williams, V.L. Moruzzi, J. Kübler and K. Schwarz Bull. Am. Phys. Soc 29 (1984) 278 (1984).

[66] K. Schwarz and P. Mohn J. Phys. F: Met. Phys 14 (1984) L129 (1984).

[67] M. Methfessel, A. T. Paxton, Phys. Rev. B 40 (1989) 3616.

[68] P. E. Blöchl, O. Jepsen, O. K. Andersen, Phys. Rev. B 49 (1994) 16223.

[69] J. H. Larsen, J. T. Ranney, D. E. Starr, J. E. Musgrove and C. T. Campbell Phys. Rev. B 63 (1996) 195410.

[70] C.R. Henry Surf. Sci. Rep. 31 (1998) 231.

[71] J. T. Ranney, D. E. Starr, J. E. Musgrove, D. J. Bald and C. T. Campbell, Faraday Discuss. 114 (1999) 195.

[72] D. Fuks, Y.F. Zhukovskii, E.A. Kotomin and D.E. Ellis Surf. Sci. 600 (2006) L99. 
Table 1. Total magnetization (in $\mu_{B}$ per $\mathrm{Rh}$ atom) for the $\mathrm{Rh} / \mathrm{MgO}(001)$ system for different $\mathrm{Rh}$ coverages at the optimized $\mathrm{Rh}$-surface distances. Values for isolated $\mathrm{Rh}$ layers corresponding to the three coverages are also given. The magnetization for the isolated gas-phase $\mathrm{Rh}$ atom is $1.959 \mu_{\mathrm{B}}(\mathrm{see}$ text).

Total magnetization per $\mathrm{Rh}$ atom and

optimized Rh-surface distance (in parenthesis)

\begin{tabular}{|c|c|c|c|}
\hline & $\underline{1 / 8 \mathrm{ML}}$ & $\underline{1 / 2 \mathrm{ML}}$ & $1 \mathrm{ML}$ \\
\hline Isolated Rh layer & $1.966^{\mathrm{b}}$ & $\begin{array}{l}1.685^{\mathrm{a}}, \\
1.703^{\mathrm{b}}\end{array}$ & $\begin{array}{l}1.566^{\mathrm{a}} \\
1.610^{\mathrm{b}}\end{array}$ \\
\hline \multicolumn{4}{|l|}{ Rh/MgO } \\
\hline Rh above $\mathbf{O}$ & $0.999(2.00 \AA)$ & $0.999(2.10 \AA)$ & $0.002(2.10 \AA)$ \\
\hline Rh above Mg & & $1.575 \quad(2.77 \AA)$ & $1.480(2.93 \AA)$ \\
\hline Rh above hollow & & $1.134 \quad(1.96 \AA)$ & $1.423(2.52 \AA)$ \\
\hline
\end{tabular}

${ }^{\mathrm{a} C a l c u l a t e d}$ using the $1 \mathrm{x} 1$ crystallographic cell and $8 \mathrm{MgO}$ layers, as in Fig. 1a.

${ }^{\mathrm{b}}$ Calculated using the $2 \times 2$ crystallographic cell and $4 \mathrm{MgO}$ layers, as in Fig. 1c. 
Table 2. Atom-projected spin magnetic moments (in $\mu_{\mathrm{B}}$ ) corresponding to the total magnetizations given in Table 1. The magnetic moments were calculated within standard Wigner-Seitz spheres with radii 1.402, 1.058 and $0.820 \AA$, respectively, for $\mathrm{Rh}, \mathrm{Mg}$ and $\mathrm{O}$. The magnetic moments listed for $\mathrm{Mg}$ and $\mathrm{O}$ in the table refer to those top-layer atoms which are either nearest or next-nearest neighbours of the Rh atom. All other atoms have magnetic moments close to zero.

Atom-projected spin magnetic moments (in $\left.\mu_{B}\right)$

\begin{tabular}{|c|c|c|c|c|c|c|c|c|c|}
\hline & \multicolumn{3}{|c|}{$\underline{1 / 8 \mathrm{ML}}$} & \multicolumn{3}{|c|}{$1 / 2 \mathrm{ML}$} & \multicolumn{3}{|c|}{$1 \mathrm{ML}$} \\
\hline & $\mathbf{R h}$ & Mg $_{\text {surf }}$ & $\mathbf{O}_{\text {surf }}$ & Rh & $\mathbf{M g}_{\text {surf }}$ & $\mathbf{O}_{\text {surf }}$ & Rh & $\mathbf{M g}_{\text {surf }}$ & $\mathbf{O}_{\text {surf }}$ \\
\hline Is olated Rh layer & $1.398^{\mathrm{b}}$ & & & $\begin{array}{l}1.398,{ }^{\mathrm{a}} \\
1.408^{\mathrm{b}}\end{array}$ & & & $\begin{array}{l}1.463,^{\mathrm{a}} \\
1.487^{\mathrm{b}}\end{array}$ & & \\
\hline $\mathrm{Rh} / \mathrm{MgO}$ & & & & & & & & & \\
\hline Rh above $O$ & 0.882 & -0.001 & 0.049 & 0.933 & -0.002 & 0.003 & 0.000 & 0.000 & 0.000 \\
\hline Rh above Mg & & & & 1.310 & -0.001 & 0.018 & 1.357 & -0.001 & 0.027 \\
\hline Rh above hollow & & & & 0.998 & -0.003 & 0.034 & 1.286 & -0.002 & 0.049 \\
\hline
\end{tabular}

${ }^{a}$ Calculated using the $1 \mathrm{x} 1$ crystallographic cell and $8 \mathrm{MgO}$ layers, as in Fig. $1 \mathrm{a}$.

${ }^{\mathrm{b}}$ Calculated using the $2 \times 2$ crystallographic cell and $4 \mathrm{MgO}$ layers, as in Fig. 1c. 


\section{Figure captions}

Fig 1. (a) The surface coverage definitions for the $\mathrm{Rh} / \mathrm{MgO}(001)$ systems, as seen "from above" (i.e. along $z$ ). The crosses mark the adsorption sites of $\mathrm{Rh}$ (always $\mathrm{O}$ in the example in the figure). (b) The supercell shown consists of $1 \mathrm{x} 1$ crystallographic unit cells in the $x$ and $y$ directions, and the coverage illustrated is 1 ML. (c) The supercell shown consists of $1 \mathrm{x} 1$ crystallographic unit cells in the $x$ and $y$ directions, and the coverage illustrated is 1/2 ML. (d) The supercell shown consists of 2x 2 crystallographic unit cells in the $x$ and $y$ directions, and the coverage illustrated is 1/8 ML. The lines denoted P1, P2 and P3 define the sections used for the various electron density and spin density plots.

Fig 2. DOS for bulk $\mathrm{Rh}$ and isolated Rh layers (exactly corresponding to the various, slighly buckled, optimized adlayer geometries resulting from the $\mathrm{Rh} / \mathrm{MgO}(001)$ calculations). The solid and dashed lines represent the $N_{\uparrow}(\varepsilon)$ and $N_{\downarrow}(\varepsilon)$ (majority and minority) spin components, respectively.

Fig 3 The relative energy of the $\mathrm{Rh} / \mathrm{MgO}(001)$ system ( $1 \mathrm{ML}$ on $\mathrm{O}$ ) as a function of the system magnetization, calculated for a Rh-O distance of $2.10 \AA$.

Fig 4 Total magnetization of the $\mathrm{Rh} / \mathrm{MgO}$ system as a function of the interlayer distance between the $\mathrm{Rh}$ overlayer and the surface for (a) different adsorption sites of $1 \mathrm{ML}$ coverage and (b) different coverages at the $\mathrm{O}$ site. See section 3.3.2 for a detailed discussion of the error bars.

Fig 5. Two energy-related and three electron-density related properties for $\mathrm{Rh} / \mathrm{MgO}(001)$ plotted as a function of the Rh-O distance, for adsorption of $1 \mathrm{ML}$ of Rh on top of O. Starting from the optimized geometry, the distance between the Rh layer and the oxide slab was varied, keeping their respective geometries fixed. For the DOS and Rh projected DOS, the majority and minority spin components are shown, in states $/ \mathrm{eV}$. The total electron density, the difference electron density, i.e. $\Delta \rho(\mathbf{r})=\rho(\mathrm{Rh} / \mathrm{MgO})-[\rho(\mathrm{Rh}-$ layer in the same geometry $)+[\rho(\mathrm{MgO}-\mathrm{slab}$ in the same geometry $)]$ and the spin density, $\sigma(\mathbf{r})$, are shown as contour plots, where solid and dashed contour lines represented electron excess and electron loss, respectively. The contour lines start at \pm 0.0005 e/a.u., increasing by a factor of $10^{0.2}$ for every contour line, i.e. they are at $0.0034,0.0053$, $0.0085,0.0134,0.0213,0.0338,0.0535,0.0848,0.1344,0.2130,0.3377,0.5352,0.8483$, $1.3445, \ldots \mathrm{e} / \AA^{3}$, and correspondingly for the negative contours. 
Fig 6. The same types of plots as in Fig. 5 , but for $1 / 2$ ML Rh coverage on the $\mathrm{O}$ sites.

Fig 7. The same types of plots as in Fig. 5, but for 1/8 ML Rh coverage on the O sites.

Fig 8. The same types of plots as in Fig. 5, but for $1 \mathrm{ML}$ Rh coverage on the Mg sites.

Fig 9. The same types of plots as in Fig. 5, but for 1 ML coverage of Rh on the "hollow" sites.

Fig 10. Schematic magnetic phase diagram for the $\mathrm{Rh} / \mathrm{MgO}(001)$ system. This shows our calculated regions of localized and itinerant magnetism and paramagetism, plotted as a function of the Rh-Rh and Rh-surface interaction strengths (see text for details). The diagram includes results from the calculations for all the different coverages and interaction sites at the optimal layer-slab distance for each case. 
Figure(s)

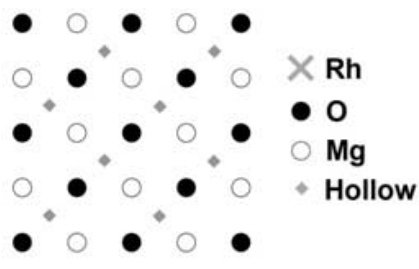

(a) Adsorption sites

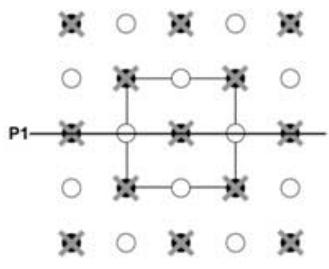

(b) $1 \mathrm{ML}$

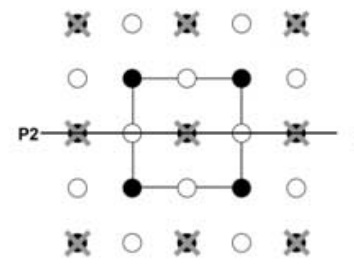

(c) $1 / 2 \mathrm{ML}$

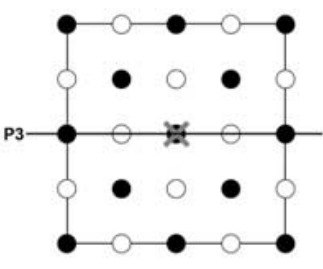

(d) $1 / 8 \mathrm{ML}$

Fig 1 


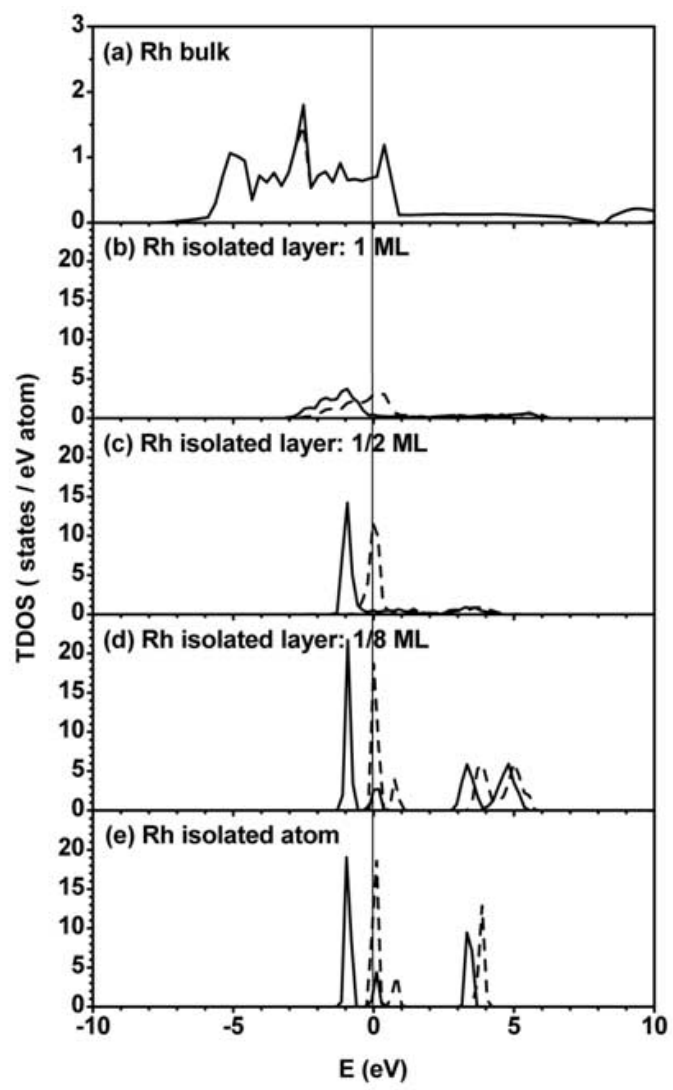

Fig 2 


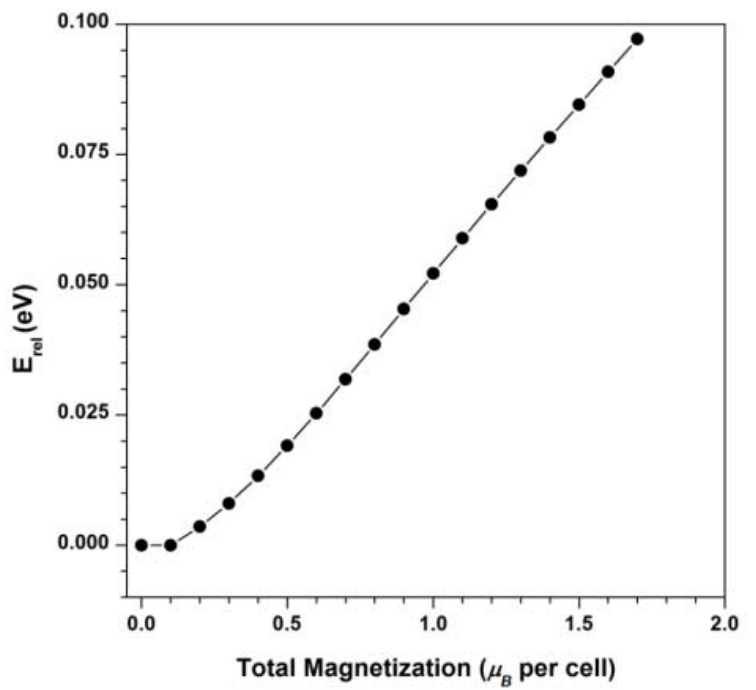

Fig 3

- 3 - 


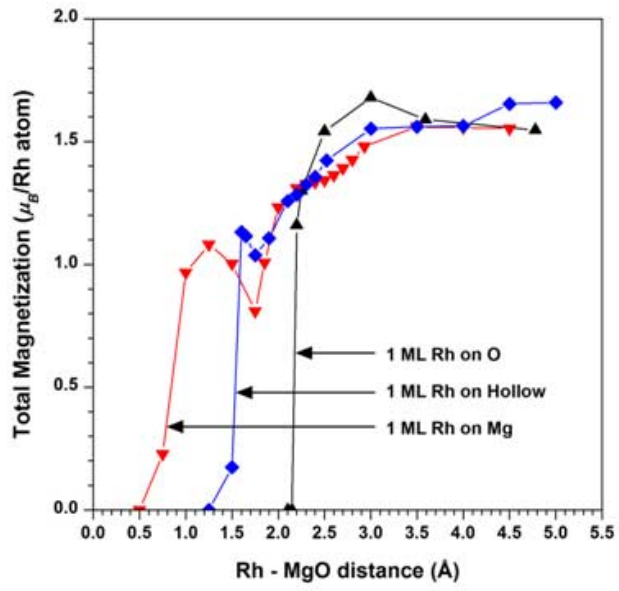

(a)

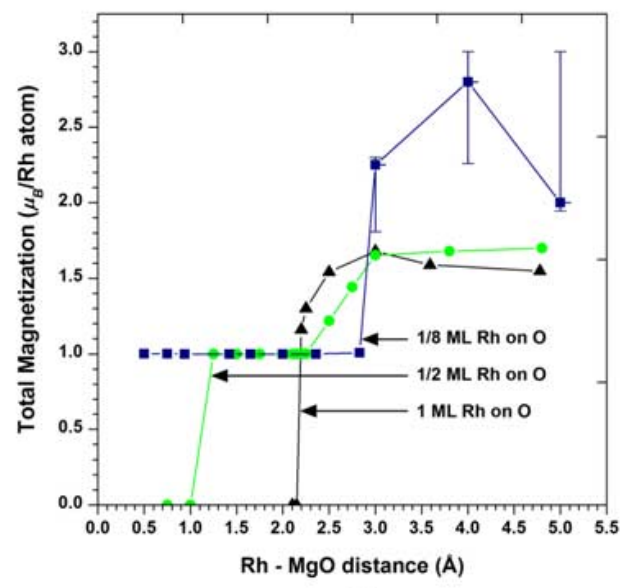

(b)

Fig 4 

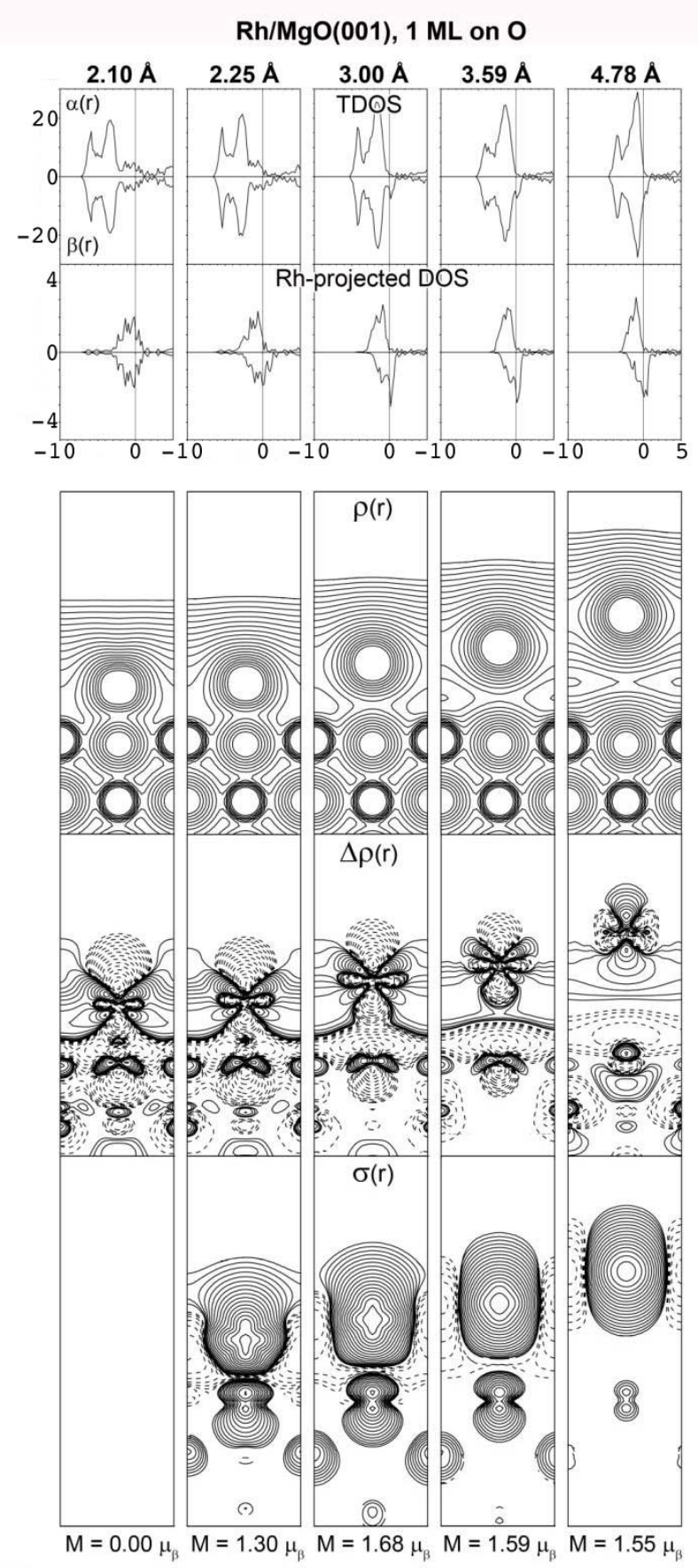

Fig 5 


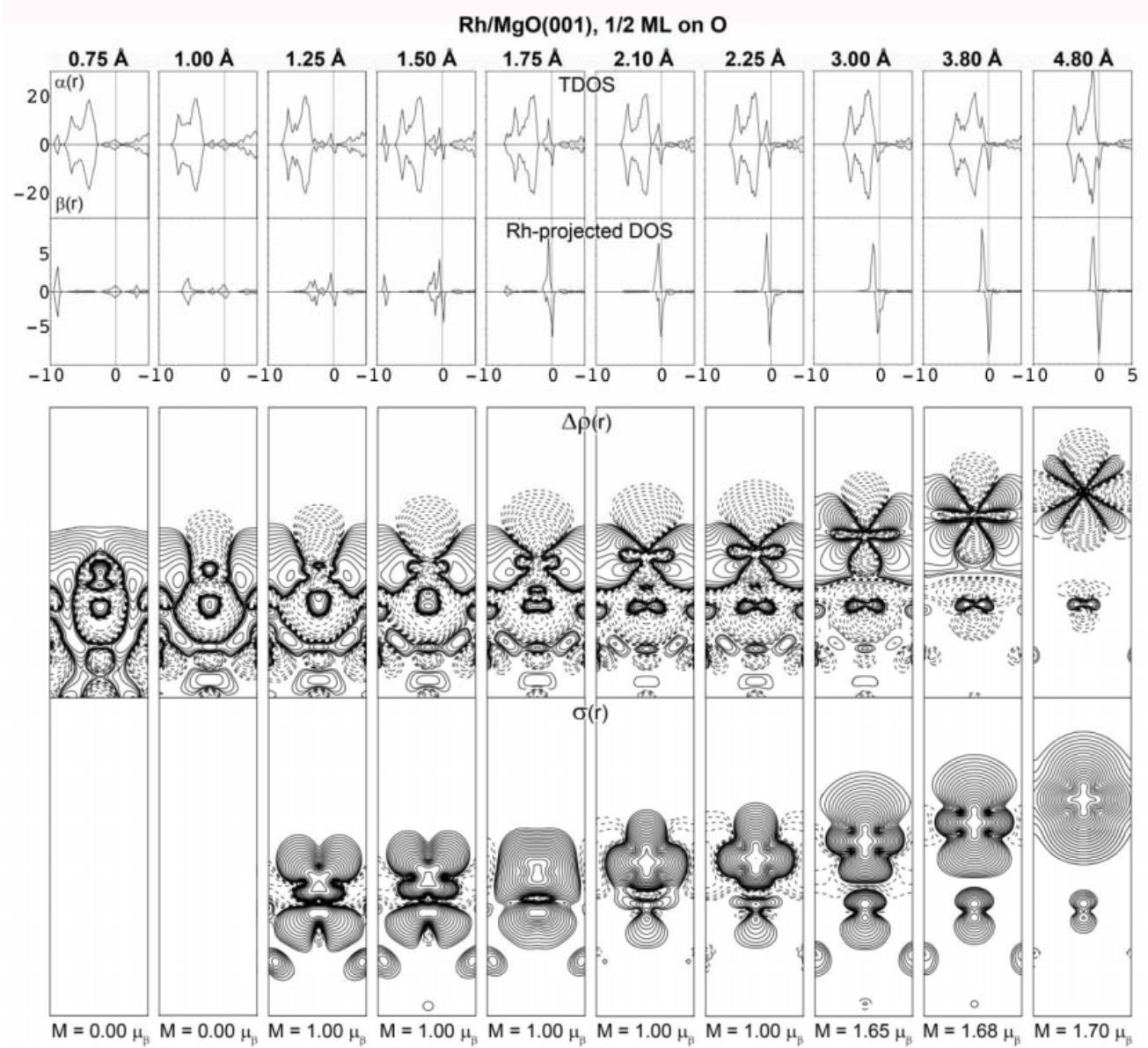

Fig 6 
$\mathrm{Rh} / \mathrm{MgO}(001), 1 / 8 \mathrm{ML}$ on $\mathrm{O}$
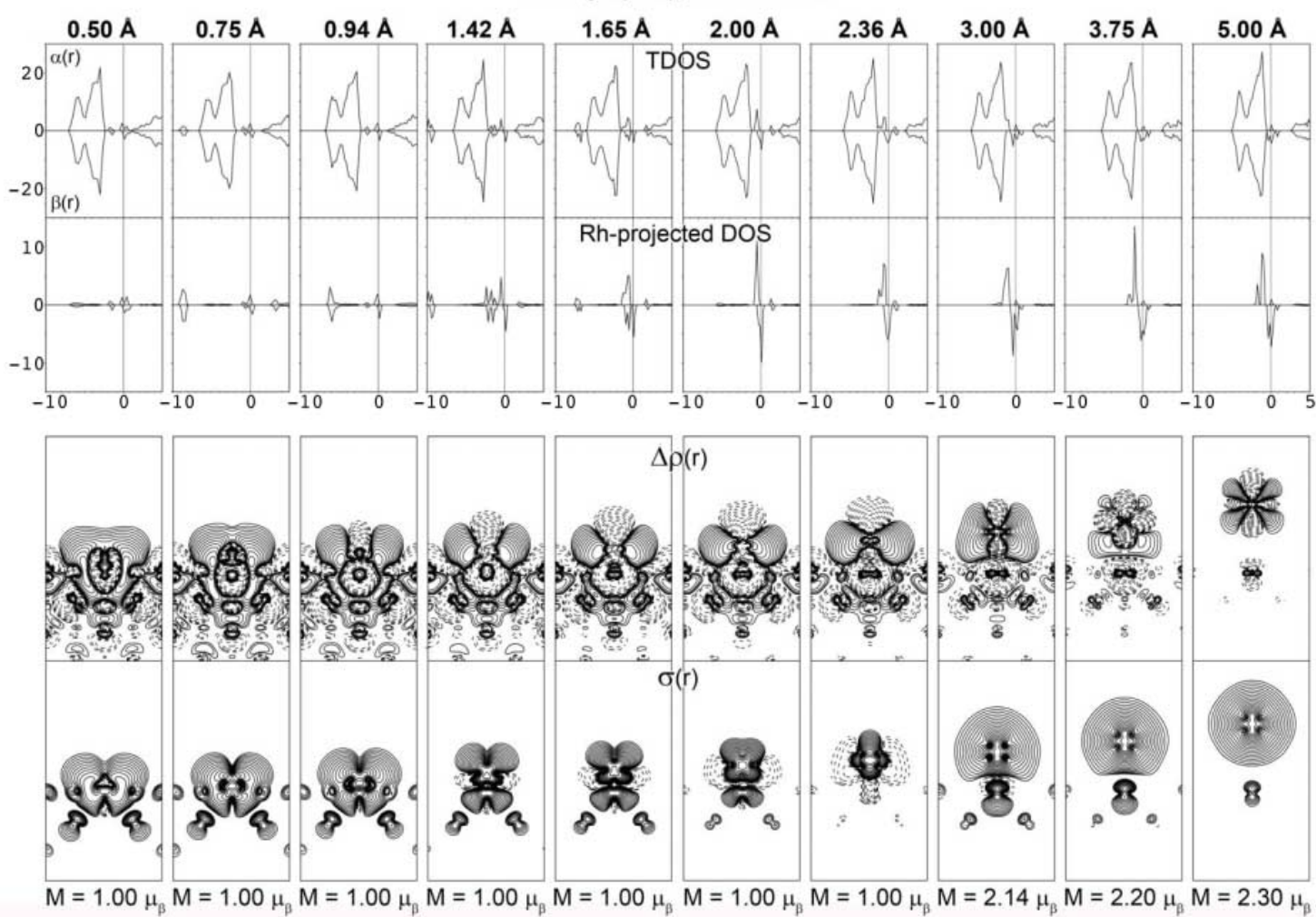

Fig 7 
$\mathrm{Rh} / \mathrm{MgO}(001), 1 \mathrm{ML}$ on $\mathrm{Mg}$
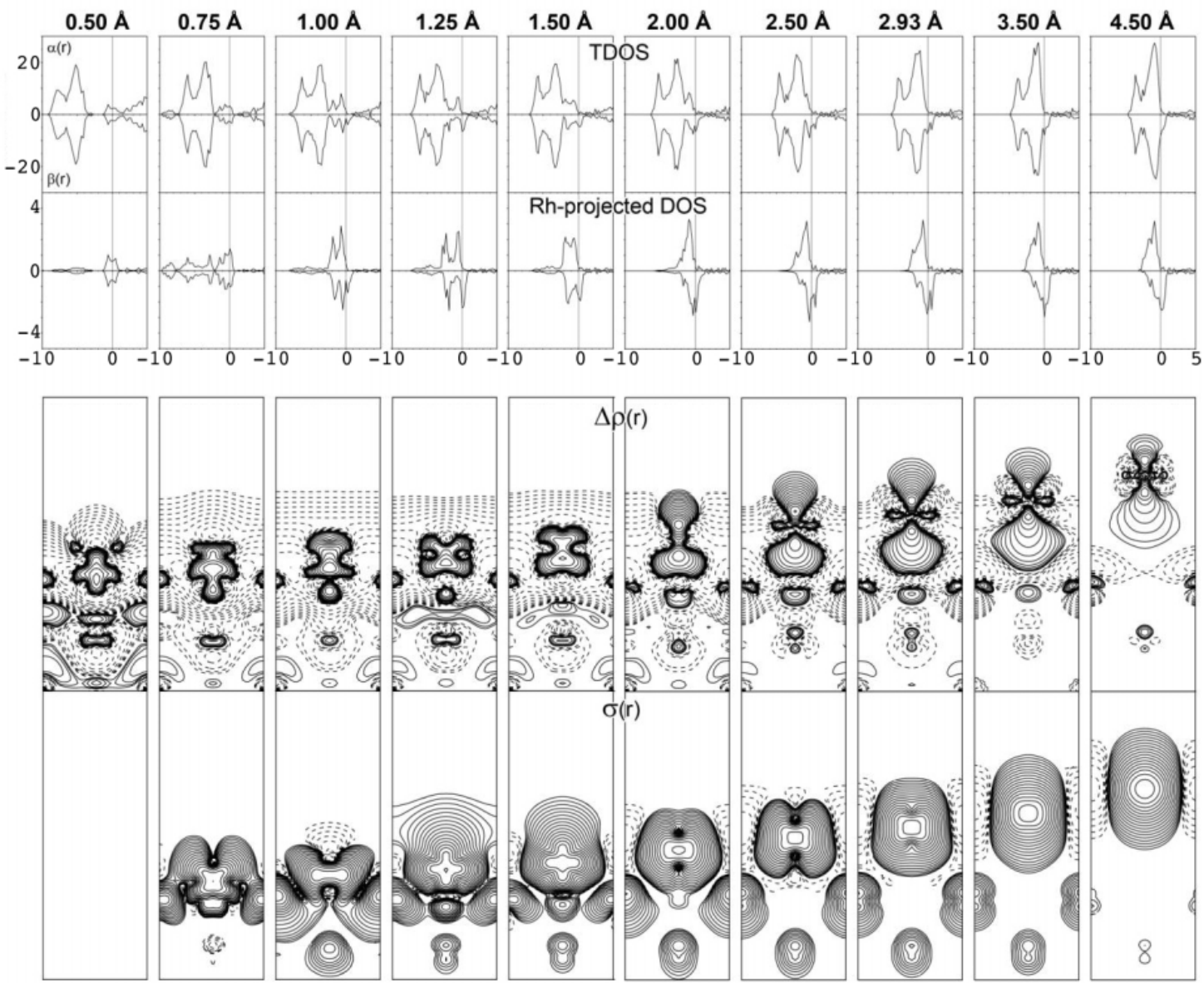

$M=0.00 \mu_{\beta} \quad M=0.23 \mu_{\beta} \quad M=0.97 \mu_{\beta} \quad M=1.08 \mu_{\beta} \quad M=1.00 \mu_{8} \quad M=1.23 \mu_{\beta} \quad M=1.34 \mu_{8} \quad M=1.48 \mu_{8} \quad M=1.56 \mu_{8} \quad M=1.56 \mu_{\beta}$

Fig 8 
바잉ำ

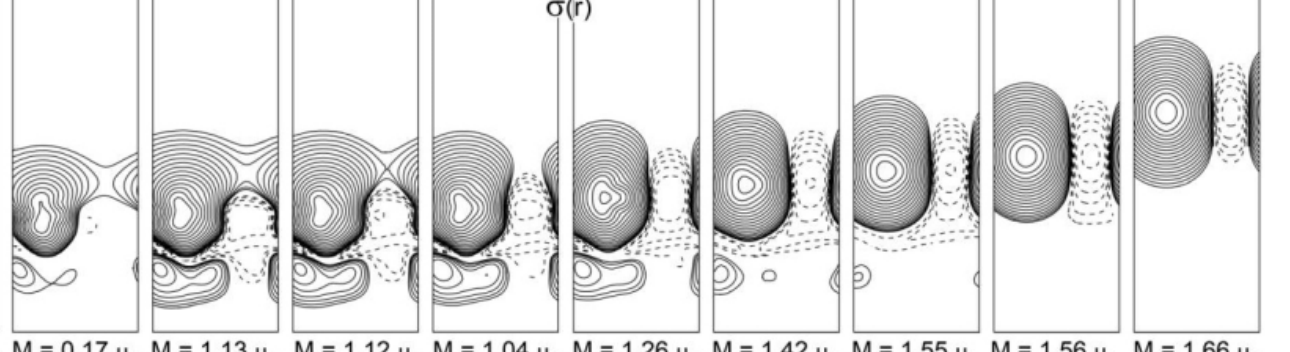

Fig 9 
a)

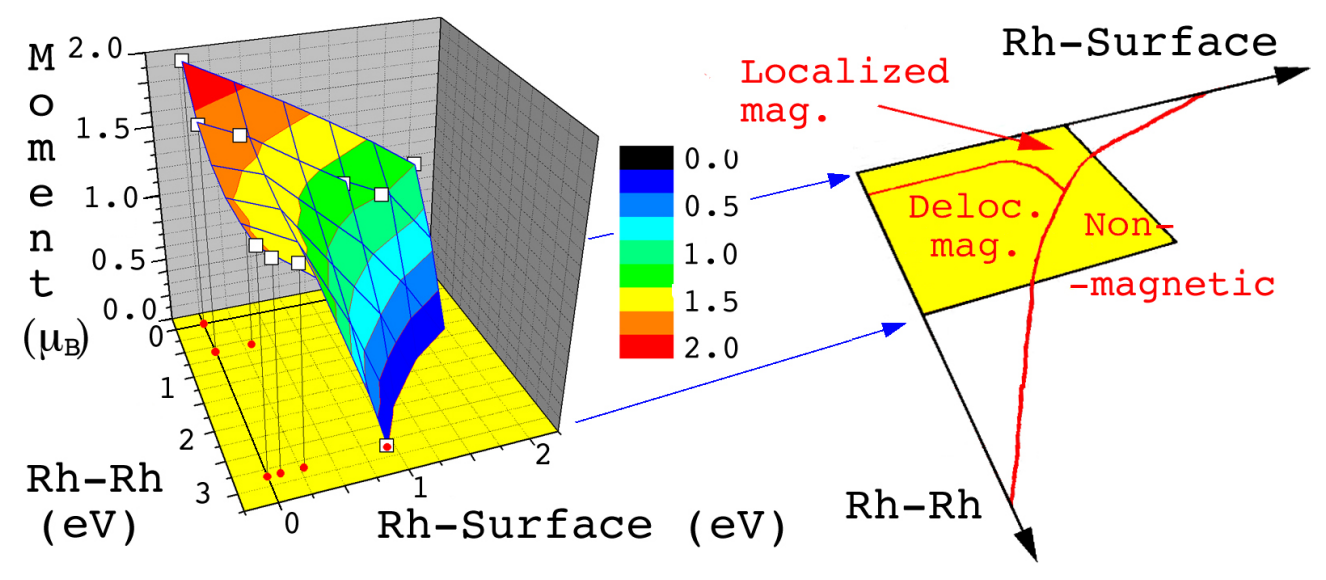

Fig 10 b) 